\title{
Desenvolvimento de um Banco de Dados Geográficos para a Análise Espacial da Lashimaniose Visceral em Cametá-PA
}

\author{
Cláudia Souza ${ }^{1}$, Douglas Gasparetto ${ }^{2}$, Cleice T. N. Souza ${ }^{3}$, Nelson Soffiatti ${ }^{4}$, \\ Roberto Brandão ${ }^{5}$, Nelson Veiga ${ }^{6}$ \\ ${ }^{1}$ Instituto Evandro Chagas, Laboratório de Geoprocessamento, \\ claudiacnsouza@yahoo.com.br \\ ${ }^{2}$ Instituto Evandro Chagas, Laboratório de Geoprocessamento, \\ douglaslabgeo@gamil.com \\ ${ }^{3}$ Centro Universitário do Estado do Pará, Instituto Evandro Chagas, \\ Laboratório de Geoprocessamento, cleicetnsouza@yahoo.com.br \\ ${ }^{4}$ Instituto Evandro Chagas, Laboratório de Geoprocessamento, \\ nelsonsoffiatti@iec.pa.gov.br \\ ${ }^{5}$ Instituto Evandro Chagas, Laboratório de Geoprocessamento, \\ rcfbmc@yahoo.com.br \\ ${ }^{5}$ Instituto Evandro Chagas, Laboratório de Geoprocessamento, \\ necoveiga@uol.com.br
}

Resumo. Neste artigo é mostrado o desenvolvimento de um banco de dados geográfico para subsidiar a análise da distribuição espacial da incidência da Leshimaniose Visceral no município de Cametá, no estado do Pará. Para tal objetivo desenvolveu-se um banco de dados geográfico com dados ecoepidemiológicos utilizando o PostGis. Após o processo do projeto lógico e físico da base de dados foi elaborado "queries" de consultas espaciais, conectadas ao PostGis, para a extração de informações. Posteriormente, utilizou-se o SIG OpenJump para a elaboração dos mapas temáticos. Como resultado observou-se que com as consultas espaciais, obtêm um rápido resultado sobre quais possíveis criadouros são propícios a proliferação da doença, utilizando as tecnologias PostGis e OpenJump, possibilitando expressar visualmente a incidência da doença de Leishimaniose Visceral no município estudado.

\section{Introdução}

A Leishimaniose Visceral (LV), também conhecida como Calazar, é uma doença parasitária cujo agente etiológico é o protozoário do gênero Leishimania, sua transmissão é feita através da picada do mosquito flebotomíneo, conhecido popularmente por mosquito palha ou birigui [Waldman e Rosa 1998]. Segundo a Organização Mundial de Saúde (OMS), as Leishmanioses afetam cerca de dois milhões de pessoas por ano, com 500 mil casos de forma visceral. É também considerada emergente em indivíduos portadores de infecção pelo vírus da imunodeficiência adquirida (HIV), tornando-se umas das mais importantes doenças da atualidade [Ministério da Saúde 2004]. O registro do primeiro caso da doença no Brasil ocorreu em 1913, quando Migone, no Paraguai, descreveu o caso em material de necropsia de paciente oriundo de Boa Esperança, Mato Grosso.

No âmbito epidemiológico - área do conhecimento que estuda a freqüência da distribuição ou eventos relacionados à saúde em populações específicas e a aplicação desses estudos no controle dos problemas de saúde [Waldman e Rosa 1998] - o geoprocessamento têm sido adotado como uma ferramenta de integração de dados ambientais, socioeconômicos e de saúde que permite realizar o mapeamento de doenças [Resendes et al. 2007]. 


\section{Revista TECCEN - Edição Especial - volume 2 - número 1 - março de 2009 - ISSN 1984-0993}

Nesse contexto, sistemas de informação e banco de dados têm sido utilizados em diversos estudos como suporte a dados geográficos. Logo, Sistemas de Informação Geográficos (SIGs) e Banco de Dados Geográficos (BDGs) quando integrados possibilitam a análise, manipulação e recuperação de dados geográficos.

Entre as tecnologias de Sistemas Gerenciadores de Banco de Dados Espaciais (SGBDE), aplicadas ao contexto de software livre (SL), o módulo de extensão espacial PostGis do PostGreSQL foi adotado neste estudo, por apresentar uma grande quantidade de funções espaciais.

Além da seção introdutória, este artigo está organizado da seguinte forma: na Seção 2 é apresentada uma descrição sobre a localização da área que está sendo estudada; na Seção 3 é detalhada a metodologia adotada no estudo; na Seção 4 são apresentados os resultados obtidos pelas análises desenvolvidas na presente pesquisa.

\section{Caracterização da Área de Estudo}

\subsection{Localização da Área de Estudo}

O Sistema de Informação de Agravos e Notificação (SINAN) apresenta um relatório gerencial a respeito de todos os casos notificados (sejam clinicamente confirmados ou não) sobre a doença LV no município de Cametá-PA. A Tabela 1 apresenta os dados retirados do sistema que correspondem aos períodos de 2007 a 2008.

Tabela 1. Relatório Gerencial de todos os casos notificados de LV, no período de 2007 a 2008, em Cametá-PA.

Fonte: SINAN

\begin{tabular}{|l|c|c|c|c|}
\hline \multirow{2}{*}{} & \multicolumn{3}{|c|}{ Leshimaniose Visceral - Cametá } \\
\cline { 2 - 5 } & $\begin{array}{c}\text { Notificados } \\
\text { pelo Município }\end{array}$ & $\begin{array}{c}\text { Notificados fora } \\
\text { do Município }\end{array}$ \\
\cline { 2 - 5 } & $\mathbf{2 0 0 7}$ & $\mathbf{2 0 0 8}$ & $\mathbf{2 0 0 7}$ & $\mathbf{2 0 0 8}$ \\
\hline Número Absoluto & 247 & 26 & 26 & 1 \\
\hline Porcentagem & $90.48 \%$ & $96.30 \%$ & $9.52 \%$ & $3.70 \%$ \\
\hline Total de Registro & 273 & 1 & & \\
\hline
\end{tabular}

Observando-se a expressividade da incidência da LV, nos períodos correspondentes, através dos registros apresentados, o povoado Enseada e os lugarejos Ajó, Vacaria e Vacajó localizados a norte e ao sul de Cametá-PA, cuja sede pertence à Mesorregião do Nordeste Paraense e a Microrregião de Cametá, foram as localidades alvos para a análise espacial da distribuição da doença. A localização geográfica da sede municipal corresponde às seguintes coordenadas: 02 ${ }^{\circ} 14^{\prime} 54^{\prime}$ ' de latitude Sul e 49³0'12' de longitude a Oeste de Greenwich. A Figura 1 apresenta o mapa temático referente à localização geográfica do município em estudo. 


\section{Revista TECCEN - Edição Especial - volume 2 - número 1 - março de 2009 - ISSN 1984-0993}

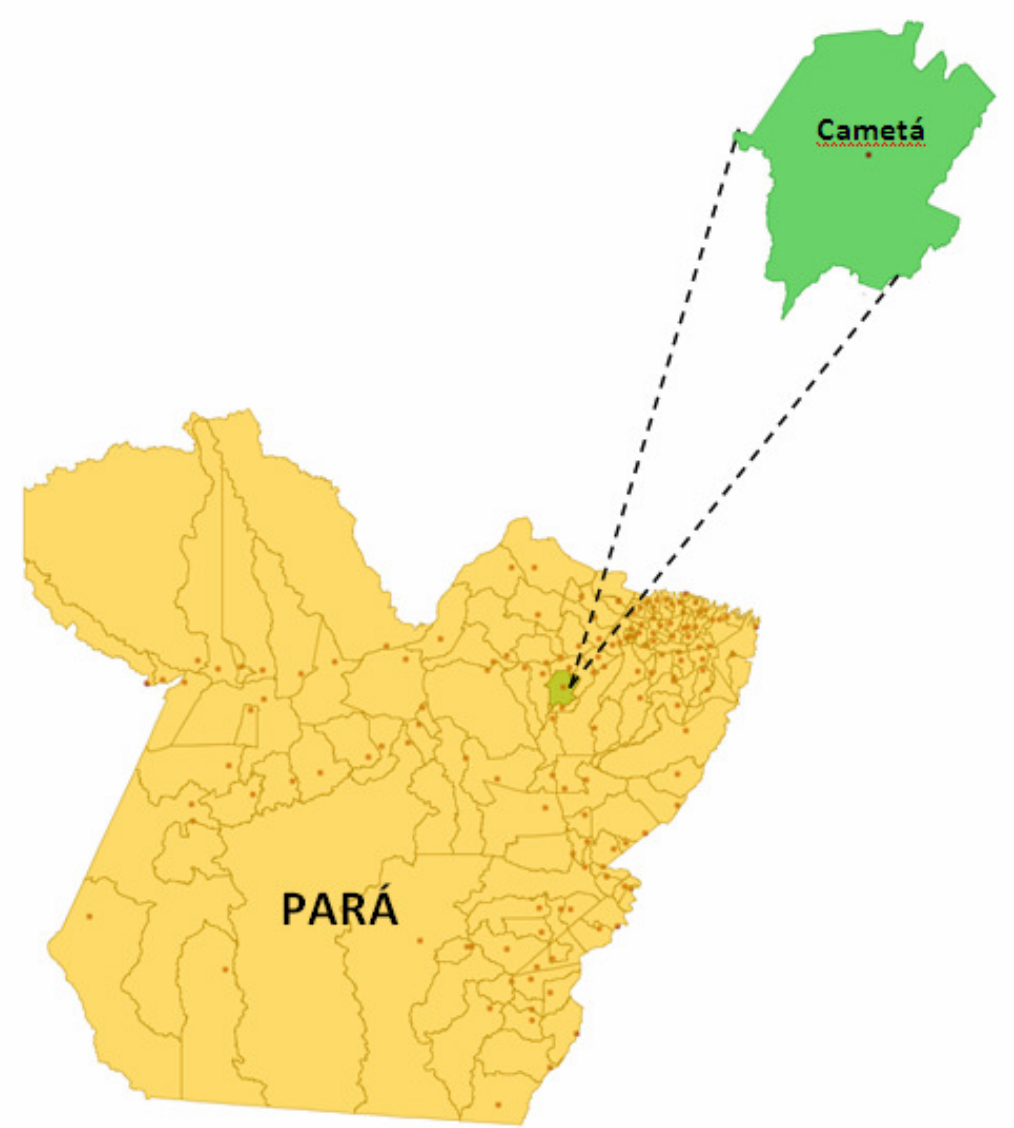

Figura 2. Mapa Temático da área de estudo: Cametá, no estado do Pará.

Fonte: Laboratório de Geoprocessamento/SVS/MS

\subsection{Aspectos Sócio-Econômicos}

O padrão de transmissão da doença de LV vem mudando ao longo dos anos, predominado pelas características ambientais, rurais e periurbanas.

Nesse sentido, baseado em levantamentos bibliográficos e questionário sócioeconômico local, percebe-se o ecletismo de subsistência das famílias, como por exemplo: cultivo de hortaliças e banana, de consumo diário nas feiras de Cametá onde a mercadoria circulante é convertida em mais-valia, desta forma, a criação de aves e suínos, prática comum entre os municípios diminui, limitando-se ao escambo entre as famílias.

Há também a criação de animais domésticos, como cães (principais reservatórios do vetor da LV), em perodomicílio, desta forma, contribuindo para a aproximação do vetor da doença, a Figura 2 demonstra as características do ambiente vivenciado pelas famílias no município em questão.

Devido a estes fatores, o caráter endêmico da LV vem se constituindo num grande desafio para as autoridades de saúde local, dada a grande mobilidade social da região. 


\section{Revista TECCEN - Edição Especial - volume 2 - número 1 - março de 2009 - ISSN 1984-0993}
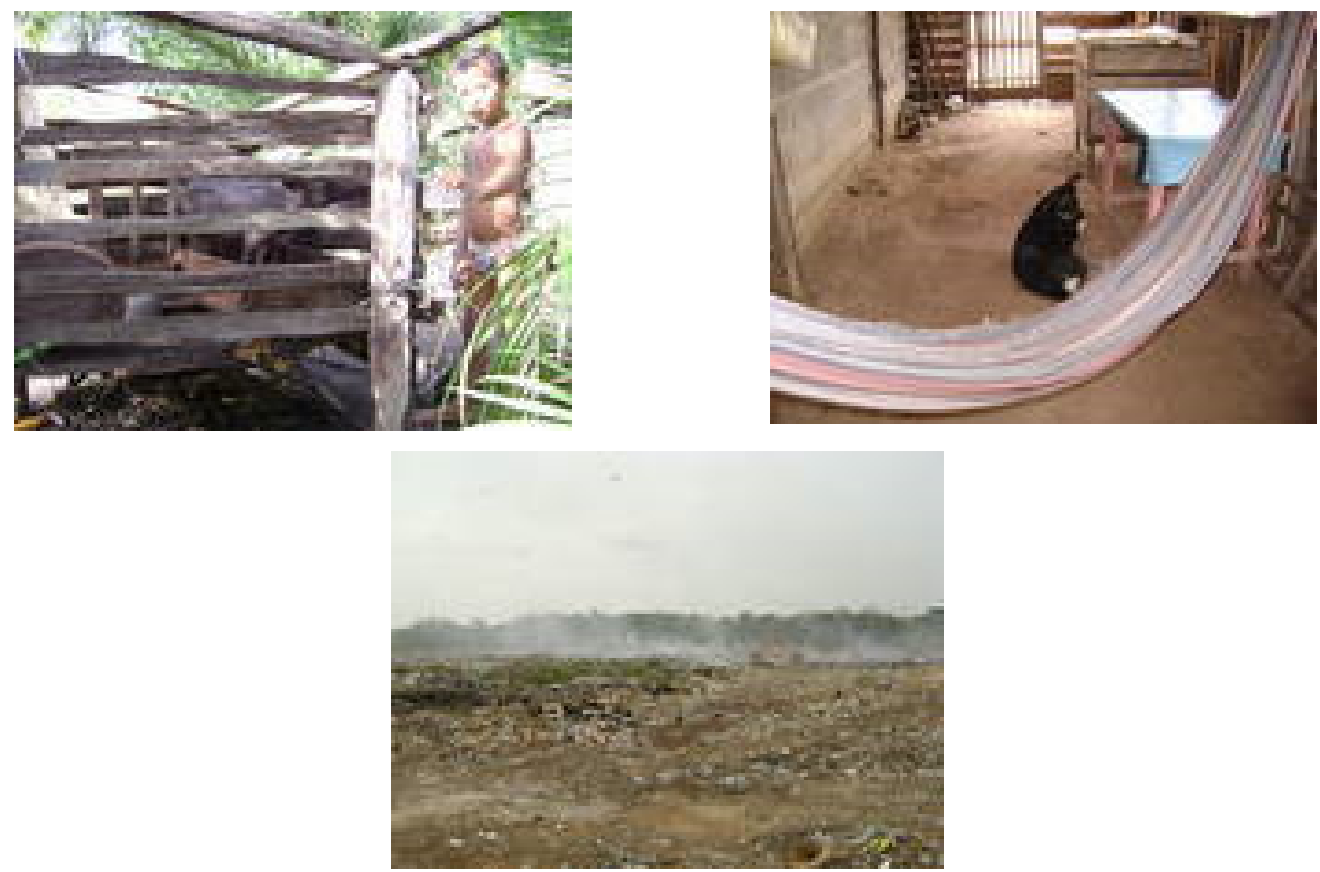

Figura 2. Aspectos Sócio-Econômicos Locais, em Cametá-Pa

Fonte: Laboratório de Geoprocessamento/SVS/MS

\section{Metodologia}

O método de pesquisa deste trabalho foi estruturado em uma sequiência de cinco etapas principais: (a) levantamento do referencial teórico sobre Vigilância em Saúde (VS) e Epidemiologia da Leshimaniose Visceral; (b) georreferenciamento de dados ecoepidemiológicos coletados em campo, utilizando o Sistema de Posicionamento Global (GPS); (c) desenvolvimento de um BDG utilizando o SGBDE Postgis e (e) realização de uma análise espacial subsidiada por mapas temáticos que expressam a distribuição espacial da incidência da Leshmaniose Visceral nas áreas de estudos preconizadas.

Nas subseções a seguir são apresentadas as geotecnologias adotadas que subsidiaram o desenvolvimento da expressão visual (mapas temáticos) que representam a relação espacial do fenômeno ecoepidemiológico que está sendo estudado.

\subsection{Sistema Gerenciador de Banco de Dados Geográficos (SGBDE): PostGis}

O PostGis é a extensão espacial do PostGreSQL, tal módulo é responsável por adicionar entidades geográficas ao seu SGBD. O PostGis possibilita armazenar, recuperar e analisar dados espaciais em um SIG [PostGis 2005].

O SGBDE apresenta um número expressivo de funções espaciais/topologias que são utilizadas para subsidiarem análises espaciais. No contexto deste trabalho, tais funções são aplicadas para a análise ecoepidemiológica referente à distribuição espacial de reservatórios, criadouros ou vetores de transmissão da doença Leshimaniose Visceral.

Nesse sentido, o artigo apresenta o processo de geração dos scripts de criação do banco de dados no PostGis. 


\section{Revista TECCEN - Edição Especial - volume 2 - número 1 - março de 2009 - ISSN 1984-0993}

1. Primeiramente, foi implementada a tabela Paciente que objetiva armazenar, por exemplo, as informações sobre os diagnósticos laboratoriais dos prováveis indivíduos com LV.

- Query: CREATE TABLE paciente (codPaciente PRIMARY KEY, localidade varchar, nome varchar, sexo varchar, idade number, resultado varchar);

2. Posteriormente, foi desenvolvido a tabela Pontos_Estudo. A tabela armazena registros das possíveis localidades que podem abrigar ou proliferar o vetor da doença LV.

- Query: CREATE TABLE Pontos_Estudo (gid serial PRIMARY KEY, nome_areas varchar, latitude varchar, longitude varchar, y_coord float,x_coord float);

SELECT AddGeometryColumn('Pontos_Estudo_geom','the_geom','4291','POINT',2);

A execução das principais consultas espaciais suportadas pelo PostGis são demonstrada pelo SIG, conforme abordado abaixo.

\subsection{Sistema de Informação Geográfica (SIG): OpenJump}

Conforme apresentado na Introdução, o SIG tem o intuito de realizar o armazenamento, manipulação e análises espaciais complexas contidas em bases de dados cartográficas georreferenciadas e banco de dados produzidos na estrutura relacional, como por exemplo, os ambientais e epidemiológicos [Souza et al. 2008].

O OpenJump é um SIG desenvolvido em tecnologia Java, baseado no modelo de negócios SL. O SIG permite a manipulação topológica sobre geometrias 2D, o acesso de feições no formato shapefile e possui suporte a dados armazenados no PostGis [Destro, 2007]. Nesse sentido, o SIG foi utilizado para realizar a conexão com o datastore no PostGis e as consultas espaciais.

A Figura 3(a) apresenta o construtor de consultas espaciais utilizado para a implementação da função intersection. Tal função objetiva mostrar os a localização precisa de indivíduos positivados laboratorialmente para LV. A Figura 3(b) apresenta um fragmento do mapa temático, no SIG OpenJump, da distribuição espacial dos casos confirmados de transmissão da LV.

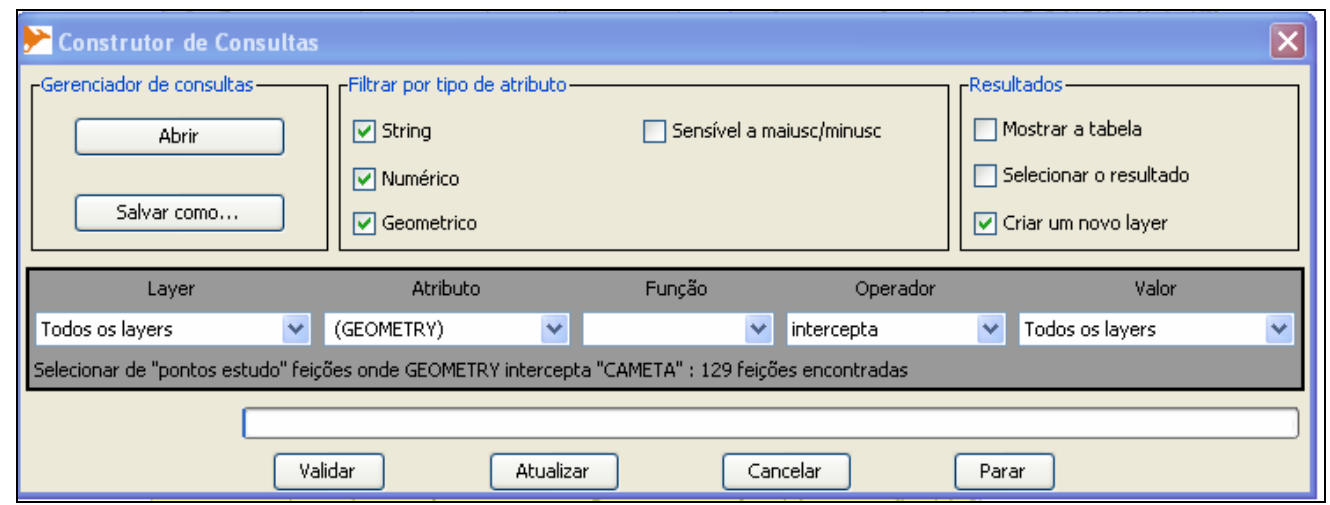

Figura 3(a). OpenJump: Construtor de Consultas Espaciais 
Revista TECCEN - Edição Especial - volume 2 - número 1 - março de 2009 ISSN 1984-0993

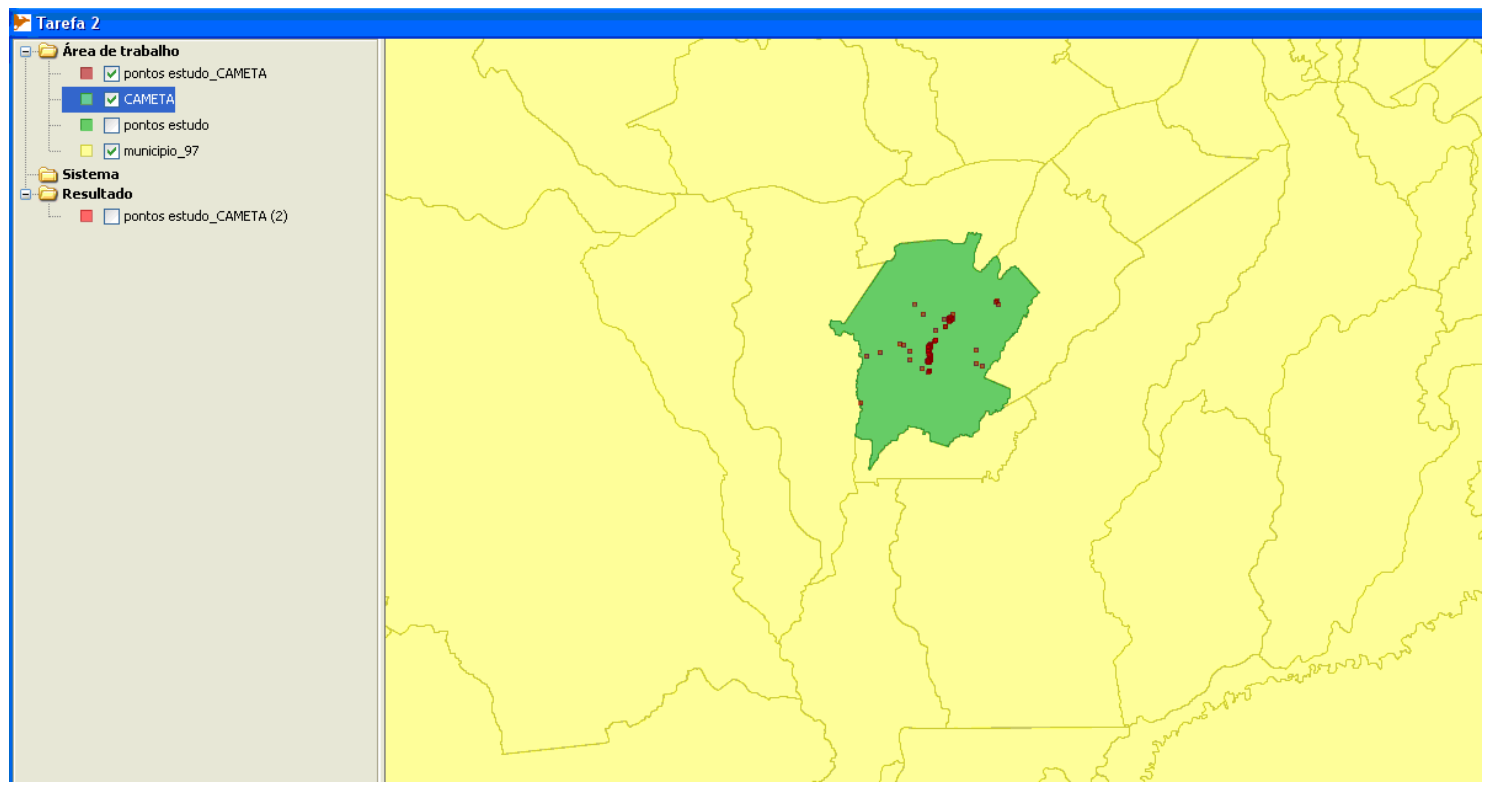

Figura 3(b). Visualização da distribuição de casos confirmados de transmissão da LV, utilizando o SIG OpenJump.

\section{Resultados e Discussões}

Como abordado na Seção 2.2, as condições sócio-econômicas das localidades de Enseada, Ajó, Vacaria e Vacajó contribuem significativamente para a aproximação do vetor da doença (flebotomíneo) em áreas de reservatórios naturais. Nesse sentido, o trabalho objetivou utilizar geotecnologias livres para o suporte das análises espaciais da distribuição da doença em Cametá, no estado do Pará.

As consultas espaciais desenvolvidas a partir das funções espaciais/topológicas do SGBDE PostGis viabilizaram análises epidemiológicas mais precisas. Tais análises foram expressas através de mapas temáticos produzidos pelo ambiente OpenJump. Ressalta-se, que o SIG apresentou um bom desempenho quando conectado ao PostGis, no sentido, de realizar consultas espaciais mais amigáveis.

Ressalta-se que tais geotecnologias, quando integradas, possibilitaram, no contexto desta pesquisa mapear os indivíduos confirmados laboratorialmente, como portadores do parasita da doença de LV.

\section{Referências Bibliográficas}

Destro, J. N.; Modelo para WEBMAP: Um estudo de Caso. 2007. 31 f. Dissertação (Mestrado em Ciências Geodésicas). Pós-Graduação em Ciências Geodésicas. Universidade Federal do Paraná. 2007.

Ministério da Saúde. Guia de Vigilância Epidemiológica ed. MS. Brasília-DF, 2004.

OpenJump. Disponível em: <http://openjump.org/wiki/show/HomePage>. Acesso em 21/01/2009.

Postgis, 2005. Disponível em:< http://postgis.refractions.net/>. Acesso em: 21/01/2009.

Resendes, Ana Paula da Costa.; Barcellos, Christovam.; Skaba, Daniel Albert.; Oliveira, Evangelina Xavier Gouveia de.; Gondim, Grácia Maria de Miranda.; Rojas, Luisa Basilia Iñiguez.; Pina, Maria de Fátima de.; Magalhães, Mônica de Avelar F.M.; Santos, Reinaldo Souza dos.; Gracie, Renata.; Santos, Simone M. Sistemas de 
Revista TECCEN - Edição Especial - volume 2 - número 1 - março de 2009 ISSN 1984-0993

Informações Geográficas e Análise Espacial na Saúde Pública. Série: Capitação e atualização em geoprocessamento em saúde. Volume 2, 2007.

Souza, C., Gasparetto, D., Souza, C.T.N, Soffiatti, N.F.L., Veiga, N. (2008). “Análise Ecoepidemiológica da Incidência da Doença de Chagas em Abaetetuba, Barcarena e Bragança, no estado do Pará, entre 2000 e 2006, utilizando Geotecnologias Livres". XV Simpósio Brasileiro de Sensoriamento Remoto, 2009.

Waldman, Eliseu Alves; Rosa, Teresa Etsuko da Costa. Vigilância em Saúde Pública. 1998. Disponível em: <http://www.saude.sc.gov.br/gestores/sala_de_leitura/ saude_e_cidadania/ed_07/index.html>. Acesso em: 10.nov.2008. 